\title{
Identification of Small-Molecule Inhibitors of FGF23 Signaling via In Silico Hot Spot Prediction and Molecular Docking to $\alpha$-Klotho
}

Shih-Hsien Liu ${ }^{*}$, Zhousheng Xiao ${ }^{2}$, Sambit K. Mishra ${ }^{3}$, Julie C. Mitchell ${ }^{3}$, Jeremy C. Smith ${ }^{1,4}$, L. Darryl Quarles ${ }^{2}$, Loukas Petridis ${ }^{1,4}$

${ }^{1}$ UT/ORNL Center for Molecular Biophysics, Oak Ridge National Laboratory, Oak Ridge, Tennessee 37831, United States

${ }^{2}$ Department of Medicine, College of Medicine, University of Tennessee Health Science Center, Memphis, Tennessee 38163, United States

${ }^{3}$ Biosciences Division, Oak Ridge National Laboratory, Oak Ridge, Tennessee 37831, United States

${ }^{4}$ Department of Biochemistry and Cellular and Molecular Biology, University of Tennessee, Knoxville, Tennessee 37996, United States

*Corresponding author :

Email: lius@ornl.gov

Phone: +1-865-241-5175

This manuscript has been authored by UT-Battelle, LLC under Contract No. DE-AC0500OR22725 with the U.S. Department of Energy. The United States Government retains and the publisher, by accepting the article for publication, acknowledges that the United States Government retains a nonexclusive, paid-up, irrevocable, worldwide license to publish or reproduce the published form of this manuscript, or allow others to do so, for United States Government purposes. The Department of Energy will provide public access to these results of federally sponsored research in accordance with the DOE Public Access Plan (https://www.energy.gov/downloads/doe-public-access-plan). 


\begin{abstract}
Fibroblast growth factor 23 (FGF23) is a therapeutic target for treating hereditary and acquired hypophosphatemic disorders, such as X-linked hypophosphatemic (XLH) rickets and tumor-induced osteomalacia (TIO), respectively. FGF23-induced hypophosphatemia is mediated by signaling through a ternary complex formed by FGF23, FGF receptor (FGFR), and $\alpha$-Klotho. Currently, disorders of excess FGF23 are treated with an FGF23-blocking antibody, Burosumab. Small-molecule drugs that disrupt protein:protein interactions necessary for the ternary complex formation offer an alternative to disrupt FGF23 signaling. In this study, the FGF23: $\alpha-$ Klotho interface was targeted to identify small-molecule protein:protein interaction inhibitors. We computationally identified "hot spots" in the FGF23: $\alpha$-Klotho interface of the ternary complex and performed in silico docking of $\sim 5.5$ million compounds from the ZINC database to the interface region of $\alpha$-Klotho from the ternary crystal structure. Following docking, 23 and 18 compounds were chosen based on the lowest binding free energies to $\alpha$-Klotho and the largest number of contacts with Tyr433, a predicted hot spot, respectively. 5 compounds available were assessed experimentally by their FGF23-mediated extracellular signal-regulated kinase (ERK) activities in vitro, and two of these reduce activities significantly. Both these compounds have a favorable predicted binding affinity, but not a large number of contacts with the hot spot residues. ZINC12409120 was found experimentally to reduce FGF23-mediated ERK activities by $70 \%$ and have a half maximal inhibitory concentration $\left(\mathrm{IC}_{50}\right)$ of $5.0 \pm 0.23 \mu \mathrm{M}$.

ZINC12409120 exhibits contacts with residues on KL1 and KL2 domains and on the linker between the two domains of $\alpha$-Klotho in in silico binding poses, thereby possibly disrupting the regular function of $\alpha$-Klotho and impeding FGF23 binding. ZINC12409120 is a candidate for lead optimization.
\end{abstract}

Keywords: FGF23, $\alpha$-Klotho, protein:protein interaction, X-linked hypophosphatemic rickets, tumor-induced osteomalacia, hot spot, molecular docking. 


\section{Introduction}

Fibroblast growth factor 23 (FGF23) is a bone-derived hormone that regulates phosphate and vitamin D homeostasis by forming a ternary complex with FGF receptor (FGFR) and $\alpha$-Klotho in renal proximal tubules. ${ }^{1,2}$ Excess FGF23 impairs phosphate reabsorption and vitamin D production, causing hereditary and acquired hypophosphatemic disorders, such as X-linked hypophosphatemic (XLH) rickets and tumor-induced osteomalacia (TIO), respectively. ${ }^{3}$

In the past, treatment for XLH consisted of phosphate and vitamin D supplements, which can cause excess phosphate and vitamin D and nephrocalcinosis. ${ }^{4,5}$ TIO can often be cured by resection of the FGF23-producing tumor, but the tumor removal is only successful in $\sim 50 \%$ of the patients. ${ }^{6,7}$ Recently, the antibody Burosumab has been approved by Food and Drug Administration (FDA) to treat XLH and TIO. Burosumab binds to and blocks FGF23 activation of the FGFR: $\alpha$-Klotho complex, ${ }^{8,9}$ and increases serum phosphate levels in patients with XLH without reported toxicity, ${ }^{10}$ but it has a long 16-day biological half-life. ${ }^{11}$

Small-molecule drugs are a promising alternative to Burosumab because of their potential oral availability and lower cost. We previously used high-throughput virtual screening and ensemble docking to the N-terminal domain of FGF23 (Protein Data Bank (PDB) code: 2P39) ${ }^{12}$ to identify a compound ZINC13407541 (N-[[2-(2-phenylethenyl)cyclopenten-1yl]methylidene]hydroxylamine), which was designed to bind to FGF23 and experimentally verified to inhibit $\alpha$-Klotho-dependent FGF23 signaling with a half maximal inhibitory concentration $\left(\mathrm{IC}_{50}\right)$ of $0.45 \pm 0.24 \mu \mathrm{M} .{ }^{13}$ Further development of a series of analogs based on ZINC13407541 structure-activity relationships identified two more compounds with enhanced drug-like properties,13a [(E)-2-((E)-4-methylstyryl)benzaldehyde oxime] and 8n [(E)-2-(4-(tertbutyl)phenyl)cyclopent-1-ene-1-carbaldehyde oxime] that blocked FGF23 signaling in vitro and significantly increased serum phosphate and vitamin D concentrations in the mouse model of $\mathrm{XLH}($ Hyp $) .{ }^{14}$

Developing small-molecule drugs disrupting the FGF23:FGFR: $\alpha$-Klotho complex is a promising strategy towards discovering novel inhibitors. Recently, the crystal structure of the ternary complex of FGF23:FGFR1c ectodomain: $\alpha$-Klotho ectodomain 1:1:1 (PDB code: 5W21) was obtained (Figure 1), ${ }^{15}$ which shows that $\alpha$-Klotho tethers the C-terminal tails of FGF23 and FGFR1c simultaneously and that $\alpha$-Klotho acts as a non-enzymatic molecular scaffold for 
FGF23 signaling. The crystal structure provides the structural information needed for computationally exploring small molecules that disrupt the protein:protein interactions/interfaces (PPIs) to reduce FGF23 signaling.

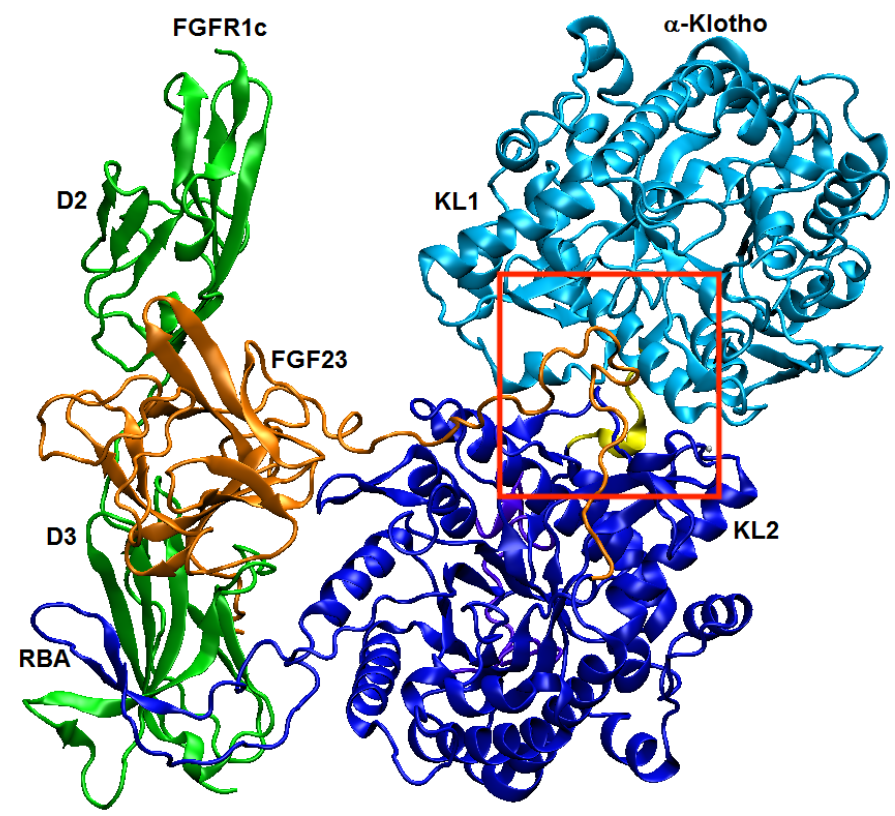

Figure 1. The crystal structure of FGF23:FGFR1 $c^{\text {ecto }}: \alpha-K_{\text {Klotho }}{ }^{\text {ecto }} 1: 1: 1$ ternary complex (PDB code: 5W21). ${ }^{15} \mathrm{KL} 1$ and KL2 domains of $\alpha$-Klotho are light and dark blue respectively, the linker between KL1 and KL2 is yellow, and receptor-binding arm (RBA) of $\alpha$-Klotho is dark blue. FGF23 is orange with its C-terminal tail in KL1-KL2 region. D2 and D3 domains of FGFR1c are green. The red box shows the region where molecular docking to $\alpha$-Klotho was performed.

In silico screening of virtual compound libraries is a promising way to identify effective PPI inhibitors for subsequent experimental validation, ${ }^{16,17}$ and a critical step for the success of this approach is to select appropriate binding sites to dock the compounds (ligands) to. Promising ligand-binding sites in this scenario would be "hot spot" residues. These residues confer a disproportionate amount of the binding energy at the PPI, and may be druggable by small molecules. ${ }^{18}$ Hot spots can be predicted computationally, ${ }^{19-27}$ and here we use the KFC2a method, ${ }^{26}$ which is based on a machine learning predictive model and recognizes structural features of PPI. 
Here, we used the ternary crystal structure (PDB code: $5 \mathrm{~W} 21)^{15}$ to computationally identify the FGF23: $\alpha$-Klotho interface as one with druggable ligand-binding sites on $\alpha$-Klotho and with the largest fraction of hot spots. $\sim 5.5$ million lead-like compounds were then docked computationally to an isolated $\alpha$-Klotho crystal structure near its interface with FGF23 (See the red box in Figure 1). 23 and 18 compounds with the highest protein-ligand binding affinities to $\alpha$-Klotho and with the largest number of contacts with Tyr433, a promising hot spot, were identified, respectively. 5 of the 41 compounds were selected for an in vitro assay based on their availability. We found that ZINC12409120 (3,4-dihydro-1H-isoquinolin-2-yl-[3-(1H-indol-4-yl)1,2,4-oxadiazol-5-yl]methanone) reduces FGF23-mediated extracellular signal-regulated kinase (ERK) activities, one of the critical pathways for FGF23 signal transduction, ${ }^{13,28}$ by $70 \%$, and it has an $\mathrm{IC}_{50}$ of $5.0 \pm 0.23 \mu \mathrm{M}$. Further examination of ZINC12409120: $\alpha$-Klotho in silico binding poses shows that ZINC12409120 is predicted to interact with residues on KL1, KL2, and the linker between KL1 and KL2 domains, but not with a hot spot, and potentially disrupts the function of $\alpha$-Klotho and deters FGF23 binding. More compounds will be tested and more functional assays of ZINC12409120 will be conducted in future studies.

\section{Methods}

\section{Hot Spot Analysis}

We used the crystal structure of FGF23:FGFR1 $c^{\text {ecto }}: \alpha-K_{\text {Klotho }}{ }^{\text {ecto }} 1: 1: 1$ ternary complex (PDB code: 5W21). ${ }^{15}$ The four missing residues (Glu957-Glu960) on the C-terminal tail of $\alpha$-Klotho were patched using MODELLER, ${ }^{29}$ and the missing atoms within each residue on the complex were then built using VMD. ${ }^{30}$ Protonation states were determined using CHARMM36 force field $^{31,32}$ and GROMACS. ${ }^{33,34}$ The complex for docking was prepared using MGLTools ${ }^{35}$ (https://ccsb.scripps.edu/mgltools) to remove its non-polar hydrogen atoms and lone pairs. This structure was uploaded to KFC Server ${ }^{26,27}$ (https://mitchell-web.ornl.gov/KFC_Server/index.php) and FTMap Server ${ }^{25}$ (https://ftmap.bu.edu) for predicting hot spots and druggable sites at the PPI, respectively. 


\section{Molecular Docking to $\alpha$-Klotho}

Docking was performed on the $\alpha$-Klotho structures extracted from the ternary complex and from snapshots selected from molecular-dynamics (MD) simulations as described below. The latter approach, known as ensemble docking, takes into account the thermal fluctuations of the binding-site atoms. ${ }^{36}$

MD simulations for ensemble docking

$\alpha$-Klotho was centered in a dodecahedron box with a $15 \AA$ padding between $\alpha$-Klotho and the box. The box was then solvated with water, and $8 \mathrm{Na}^{+}$ions were also included to neutralize the system. To ensure our findings do not depend on one initial configuration, five independent MD simulations (i.e., instances) with different initial velocity distributions were performed using the CHARMM36 force field parameters ${ }^{31,32}$ for $\alpha$-Klotho, the TIP3P model ${ }^{37}$ for water and the GROMACS simulation code. ${ }^{33,34}$

For each of the five simulations, energy minimization was performed, followed by equilibration in the NVT and then the NPT ensembles with a time step of 2 fs at $310 \mathrm{~K}$ and 1 bar for $1 \mathrm{~ns}$, with non-hydrogen atoms on the $\alpha$-Klotho restrained using a force constant of 1000 $\mathrm{kJ} / \mathrm{mol} / \mathrm{nm}^{2}$ in each dimension. Subsequently, each of the five simulations was continued for production in the NPT ensemble for $200 \mathrm{~ns}$, with only the $\mathrm{Zn}$ atom on the $\alpha$-Klotho restrained using the above force constant to maintain its coordination state. The instantaneous energies and configurations were saved every $10 \mathrm{ps}$, and the last $100 \mathrm{~ns}$ were used for clustering. The details of the simulation settings and validation of data in the last $100 \mathrm{~ns}$ are included in Supporting Information S1.

Root-mean-square deviation (RMSD)-based clustering of $\alpha$-Klotho was performed using the gromos $^{38}$ method on all non-hydrogen atoms of 9 residues at the KL1:KL2:FGF23 interface (i.e., interface residues) identified in the ternary crystal structure ${ }^{15}$ (Table 2). The cutoff of RMSD from the crystal structure was varied between 1.5 and $2.0 \AA$ so as to identify the optimal RMSD cutoff, such that the total number of clusters is $\sim 40$ and the number of 1-frame clusters is minimal. ${ }^{39}$ The RMSD cutoff chosen here was $1.52 \AA$ which resulted in 38 clusters, only one of which contained 1 frame. The ten central structures, those with the smallest average RMSD from all other structures, each from the 10 most populated clusters \#1-10 representing 98.4\% of MD frames were selected as an ensemble of $\alpha$-Klotho, named MD clusters \#1-10 (Table S1). 


\section{Preparation of receptors and ligands prior to docking}

The interface residues in each MD cluster were aligned with those in the crystal structure ${ }^{15}$ using a least squares fit. The files in PDB format of the aligned structures are included in the "alpha-Klotho.zip" as Supporting Information. For docking to the crystal structure and each of the 10 MD clusters, a ligand pose-searching box of 30 x 30 x $30 \AA$ was centered at the geometric center of Tyr432 (Table S2), a potentially druggable site (Table 2), to include the 6 computationally predicted hot spots at KL1:KL2:FGF23 interface (Tables 2 and S3).

$5,450,731$ compounds in the lead-like ${ }^{40}$ and clean (without aldehydes and thiols) subsets of the ZINC database ${ }^{41}$ (http://zinc12.docking.org/subsets/clean-leads) were used, and compounds with different protonation states but the same ZINC ID were included. Million-compound docking has been previously shown to discover protein inhibitors with nanomolar binding affinity. $^{42}$ The files for docking were prepared using MGLTools ${ }^{35}$

(https://ccsb.scripps.edu/mgltools) where non-polar hydrogen atoms and lone pairs were removed and Gasteiger partial atomic charges were not added to ligands.

\section{Three-phase docking}

In the first phase, VinaMPI, ${ }^{43}$ a modified version of AutoDock Vina ${ }^{44}$ optimized for performing calculations on supercomputers, was used to dock the 5,450,731 ZINC compounds to the $\alpha$-Klotho crystal structure ${ }^{15}$ with an exhaustiveness of 10 . For each compound, 9 binding poses were generated each with estimated protein-ligand free energy of binding $(\Delta G)$.

In the second phase, those 820,835 compounds whose $\Delta \mathrm{G}$ were within $3 \mathrm{kcal} / \mathrm{mol}$ from the $\Delta \mathrm{G}$ of the best ranked ligand were re-docked to the same region of $\alpha$-Klotho crystal structure with a larger exhaustiveness of 20 to enhance binding pose prediction. $3 \mathrm{kcal} / \mathrm{mol}$ was used as it

is the uncertainty of $\Delta \mathrm{G}$ in AutoDock Vina. ${ }^{44} 41$ compounds were chosen for further evaluation: 23 compounds were selected based on the lowest $\Delta \mathrm{G}$ from the second phase, and the other 18 compounds were selected because one of their binding poses had the largest number of nonhydrogen atomic contacts with Tyr433, a critical hot spot residue at KL1:KL2:FGF23 interface of $\alpha$-Klotho ${ }^{15}$ (Table 2). A contact here is defined as non-hydrogen interatomic distance between protein and ligand $\leq 4.0 \AA$.

In the third phase, these 41 compounds were docked to the same region of $\alpha$-Klotho crystal structure and to the $10 \mathrm{MD}$ clusters (i.e., ensemble docking, which considers protein flexibility and has proven to be useful in early drug discovery ${ }^{36}$ ) with an exhaustiveness of 30 to further 
refine binding poses. The simplified molecular-input line-entry system (SMILES) for each of the 41 compounds is included in the "ZINC-Compound.csv" as Supporting Information. For each of the 23 and 18 compounds, the binding pose with the lowest $\Delta \mathrm{G}$ from Vina and that with the largest number of contacts with Tyr433 were rescored using $K_{\text {DEEP }}{ }^{45}$ respectively, and the ligand efficiency was also derived by dividing $\Delta \mathrm{G}\left(K_{\mathrm{DEEP}}\right)$ by the number of non-hydrogen atoms in a ligand. ${ }^{46}$

\section{In Silico Ligand: $\alpha$-Klotho Interaction Analysis}

The 2D contact maps of ZINC12409120 and ZINC05326903 with $\alpha$-Klotho were plotted using LigPlot ${ }^{+}{ }^{47}$ For hydrogen-bond calculation, the maximum hydrogen-acceptor and maximum donor-acceptor distances were set at 3.0 and $4.0 \AA$, respectively. For non-bonded contact calculation, the minimum and maximum contact distances were set at 2.0 and $4.0 \AA$, respectively.

\section{In Vitro Functional Assay}

The 5 ZINC compounds were purchased from AKos Consulting and Solutions Deutschland GmbH (Lörrach, Germany) based on their availability. To test the efficacy of the 5 ZINC compounds identified computationally on FGF23-mediated activation of the FGFR1c: $\alpha$-Klotho complex, human embryonic kidney (HEK) 293T cells were cultured in Dulbecco's modified Eagle medium (DMEM) containing $10 \mathrm{wt} \%$ of fetal bovine serum (FBS) and $1 \mathrm{wt} \%$ of penicillin and streptomycin (P/S). Since HEK 293T cells expressed FGFR1c but not transcripts encoding $\alpha$-Klotho, ${ }^{13}$ they were transiently transfected with full-length human $\alpha$-Klotho along with the ERK luciferase reporter system and Renilla luciferase-null as internal control plasmid. Transfection was performed by electroporation using Lonza Cell Line Nucleofector Kit R (Walkersville, MD). After 36-hour transfection, the transfected cells were treated with: 1) empty expression vector as control; 2) FGF23 only at $1 \mathrm{nM}$; 3 ) each of the 5 ZINC compounds at 10 $\mu \mathrm{M}$ in presence of FGF23 at $1 \mathrm{nM}$; 4) ZINC12409120 in a range of $10^{-9}-10^{-4} \mathrm{M}$ in presence or absence of FGF23 at $1 \mathrm{nM}$ to obtain half maximal inhibitory concentration $\left(\mathrm{IC}_{50}\right)$. After 5 hours, the cells were lysed, and luciferase activities were measured using BioTek Synergy H4 Hybrid Multi-Mode Microplate Reader (Winooski, VT) and Promega Dual-Luciferase Reporter Assay 
System (Madison, WI) ${ }^{13}$ Three independent experiments were run for each scenario to obtain sufficient statistics. Statistical significance between FGF23 only and each of the 2 FGF23mediated ZINC compounds was evaluated by unpaired t-test, and the calculations were performed using GraphPad Prism 5.0 (San Diego, CA). The IC 50 of ZINC12409120 was obtained graphically from concentration-effect curves using GraphPad Prism 5.0 (San Diego, CA). More details are described in our previous work. ${ }^{13}$

\section{Results and Discussion}

\section{Hot Spot Analysis}

The KL1:KL2:FGF23 interface is perhaps the most promising target for small-molecule drug discovery among the 5 interfaces identified in the ternary crystal structure, ${ }^{15}$ as it has predicted druggable sites and $73 \%$ of the interface residues are predicted hot spots (Table 1). We note that $75 \%$ of the KL2:FGF23 interface residues are predicted hot spots, but all of them are on FGF23 C-terminal tail (Table S5), which is coil-like and thus unlikely to be a competent drug-binding region.

Table 1. The 5 interfaces identified in the ternary crystal structure. ${ }^{15}$ Druggable sites and hot spots were computationally predicted using FTMap ${ }^{25}$ and the KFC2a methods, ${ }^{26}$ respectively. Hot spot \% corresponds to the ratio of number of predicted hot spots to number of interface residues. Details of each interface are shown in Tables 2 and S4-S7.

\begin{tabular}{rrrr}
\hline Interface & Druggable site & Hot spot \% & Details in Table \\
\hline KL1:KL2:FGF23 & Yes & $73 \%$ & 2 \\
D3:FGF23 & Yes & $44 \%$ & S4 \\
KL2:FGF23 & No & $75 \%$ & S5 \\
D2:FGF23 & No & $64 \%$ & S6 \\
RBA:D3 & No & $50 \%$ & S7 \\
\hline
\end{tabular}

Tyr432 and Lys435 on the KL1 domain are the two most druggable sites close to KL1:KL2:FGF23 interface, and Tyr433 on the KL1 domain is the most promising predicted hot spot on $\alpha$-Klotho (Table 2). Tyr433 was identified in the ternary crystal structure as a key residue on $\alpha$-Klotho that tethers FGF23, ${ }^{15}$ which agrees with our hot-spot prediction. In addition, 
Met833, Thr834 on the KL2 domain and Pro189, Leu190, Val192, Leu193 on FGF23 C-terminal tail also have relatively high hot spot confidence scores.

Table 2. The 15 residues defining the KL1:KL2:FGF23 interface in the ternary crystal structure, ${ }^{15}$ and additional 2 residues in bold were predicted as druggable sites using FTMap. ${ }^{25}$ The hotspot identification and confidence scores were evaluated by the KFC2a method. ${ }^{26}$ Higher confidence scores suggest higher probability of being hot spots, and 11 out of 15 interface residues are predicted hot spots. N/A: not available.

\begin{tabular}{rrrrr}
\hline Protein & Residue & Number & Hot spot & Confidence score \\
\hline & Lys & 429 & Yes & 1.05 \\
& Tyr & $\mathbf{4 3 2}$ & No & $\mathbf{- 1 . 2 1}$ \\
$\alpha$-Klotho & Tyr & 433 & Yes & 1.43 \\
KL1 domain & Lys & $\mathbf{4 3 5}$ & N/A & N/A \\
& Phe & 437 & No & -0.30 \\
& Ser & 471 & N/A & N/A \\
\hline & Lys & 823 & Yes & 0.26 \\
a-Klotho & Met & 833 & Yes & 1.38 \\
KL2 domain & Thr & 834 & Yes & 1.37 \\
& Ile & 836 & Yes & 0.20 \\
& Gln & 844 & No & -0.74 \\
\hline & Asp & 188 & Yes & 0.42 \\
& Pro & 189 & Yes & 1.31 \\
FGF23 & Leu & 190 & Yes & 1.32 \\
C-terminal tail & Asn & 191 & No & -1.19 \\
& Val & 192 & Yes & 1.94 \\
& Leu & 193 & Yes & 1.96 \\
\hline
\end{tabular}

For the 7 predicted hot spots at the KL1:KL2:FGF23 interface with relatively high confidence scores (Figure 2), Tyr433 and Met833 lie on an $\alpha$-helix (Ala428-Leu447) and a $\beta$-strand (Val830-Met833) of $\alpha$-Klotho respectively, and offer prospective binding sites for the subsequent docking campaign. The other hot spots may not be good drug-binding targets, since they are located on a coil. Most reported PPI inhibitors bind strongly to a PPI with well-defined secondary structures, which exists in the unbound protein but becomes buried when PPI complex is formed. ${ }^{48}$ 


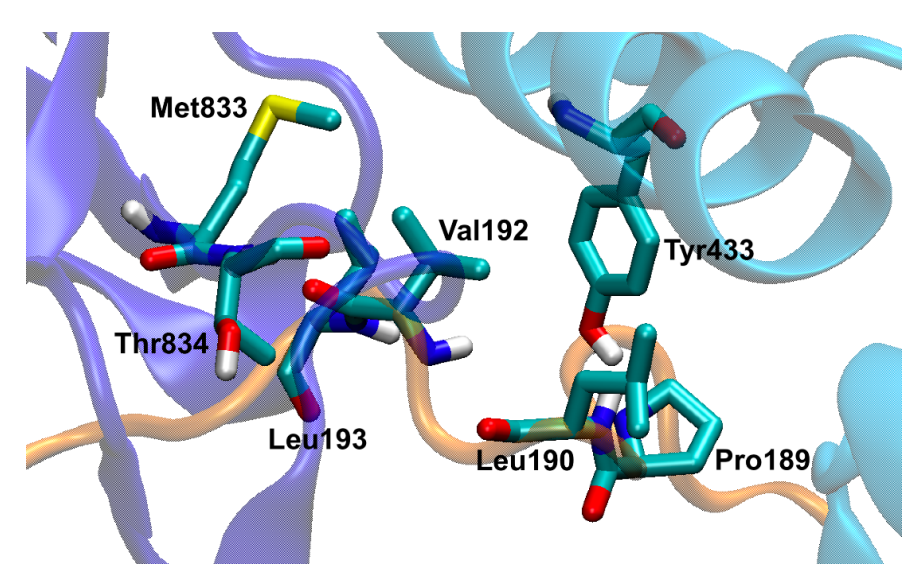

Figure 2. Visualization of the 7 calculated hot spots with relatively high confidence scores in Table 2 at KL1:KL2:FGF23 (light blue:dark blue:orange) interface of the ternary crystal structure. $^{15}$

\section{Molecular Docking to $\alpha$-Klotho}

To select compounds for experimental validation, we applied two approaches. The first approach is to identify ZINC compounds with the most favorable binding energies predicted with Vina (Table 3). 21 of the 23 compounds were predicted to bind to the $\alpha$-Klotho conformation from the crystal structure, ${ }^{15}$ while 2 bound to conformations obtained from the MD simulations. Further, $K_{\text {DEEP }}$, a protein-ligand absolute binding affinity predictor based on deep convolutional neural networks $(\mathrm{CNN}),{ }^{45}$ was used to rescore $\Delta \mathrm{G}$ for these 23 protein-ligand complexes generated by Vina. The ligand efficiency $(\eta)$ for each compound was also obtained by dividing $\Delta \mathrm{G}\left(K_{\mathrm{DEEP}}\right)$ by number of non-hydrogen atoms in a ligand to provide a useful metric for lead selection. ${ }^{46}$ The lower the value of $\eta$, the better the potency of a ligand. In addition, 21 of the 23 compounds have contacts with Tyr432 or Lys435, two druggable sites, whereas none has contact with hot spot residues at the KL1:KL2:FGF23 interface (Table S8). It is thus likely that there is no druggable cavity around these hot spots. All 23 compounds are drug-like as evaluated by SwissADME, ${ }^{49}$ and only ZINC19373000 was identified as pan assay interference compounds (PAINS). ${ }^{50} 3$ compounds (ZINC12409120, ZINC05326903, and ZINC36391530) were chosen because of their availability for an in vitro assay to test their efficacies. 
Table 3. The 23 ZINC compounds with the lowest estimated free energies of binding $(\Delta \mathrm{G})$ to $\alpha$ Klotho in the crystal-structure docking, followed by ensemble docking to refine binding poses whose receptor conformations and $\Delta \mathrm{G}$ are shown in the 2 nd and 3 rd columns, respectively. $\Delta \mathrm{G}$ rescored by $K_{\text {DEEP }}{ }^{45}$ and their corresponding ligand efficiencies $(\eta)^{46}$ are shown in the 4 th and 5 th columns, respectively. The 3 compounds in bold were selected for an in vitro assay because of their availability. The compound in red was identified as PAINS. ${ }^{50}$ Compounds with the same ID have different protonation states. N/A: not applicable.

\begin{tabular}{|c|c|c|c|c|}
\hline ZINC ID & Receptor & $\begin{array}{c}\Delta G \text { (Vina) } \\
{[\text { kcal/mol] }}\end{array}$ & $\begin{array}{r}\Delta G\left(K_{\text {DEEP }}\right) \\
{[\mathrm{kcal} / \mathrm{mol}]}\end{array}$ & $\begin{array}{r}\eta\left(K_{\text {DEEP }}\right) \\
{[\mathrm{kcal} / \mathrm{mol}]}\end{array}$ \\
\hline 12409120 & cluster $\# 8$ & -11.2 & -9.84 & -0.38 \\
\hline 70700439 & crystal & -11.1 & -8.81 & -0.34 \\
\hline 89915984 & crystal & -11.1 & -8.44 & -0.32 \\
\hline 88338906 & crystal & -11.0 & -10.60 & -0.41 \\
\hline 05089013 & crystal & -11.0 & -10.54 & -0.42 \\
\hline 05326903 & crystal & -11.0 & -10.29 & -0.41 \\
\hline 33020020 & crystal & -11.0 & -9.32 & -0.36 \\
\hline 65044553 & crystal & -11.0 & -9.11 & -0.35 \\
\hline 03908210 & cluster \#2 & -11.0 & -8.96 & -0.34 \\
\hline 98150430 & crystal & -11.0 & -8.82 & -0.34 \\
\hline 19373000 & crystal & -11.0 & -8.77 & -0.34 \\
\hline 89914674 & crystal & -11.0 & -8.34 & -0.32 \\
\hline 65236018 & crystal & -10.9 & -10.74 & -0.41 \\
\hline 69840438 & crystal & -10.9 & -9.97 & -0.38 \\
\hline 65514802 & crystal & -10.9 & -9.59 & -0.37 \\
\hline 65514802 & crystal & -10.9 & -9.58 & -0.37 \\
\hline 38747189 & crystal & -10.9 & -9.37 & -0.36 \\
\hline 36391530 & crystal & -10.9 & -9.28 & -0.36 \\
\hline 65514802 & crystal & -10.9 & -9.28 & -0.36 \\
\hline 96129233 & crystal & -10.9 & -9.15 & -0.35 \\
\hline 98150430 & crystal & -10.9 & -9.08 & -0.35 \\
\hline 89364390 & crystal & -10.9 & -8.24 & -0.33 \\
\hline 04359996 & crystal & -10.8 & -9.62 & -0.37 \\
\hline Average & $\mathrm{N} / \mathrm{A}$ & -11.0 & -9.38 & -0.36 \\
\hline
\end{tabular}

The second approach to identifying candidates for experimental validation involves 18 compounds that primarily contact the hot spot residue Tyr433 (Table 4). In addition, all these compounds have contacts with the other 3 hot spot residues at KL1:KL2:FGF23 interface (Lys429, Tyr433, and Ile836) and the druggable site (Tyr432) (Table S9), but they have less favorable $\Delta \mathrm{G}$ on average than those in Table 3 . All 18 compounds are drug-like as evaluated by 
SwissADME, ${ }^{49}$ and none was identified as PAINS. ${ }^{50} 2$ compounds (ZINC31607018 and ZINC72289860) were chosen because of their availability for an in vitro assay to test their efficacies.

Table 4. The 18 ZINC compounds whose binding poses have the largest number of nonhydrogen atomic contacts with the hot spot Tyr433 on $\alpha$-Klotho in the crystal-structure docking, followed by ensemble docking to refine binding poses whose receptor conformations, number of contacts with Tyr433, and $\Delta \mathrm{G}$ are shown in the $2 \mathrm{nd}, 3 \mathrm{rd}$, and 4 th columns, respectively. $\Delta \mathrm{G}$ rescored by $K_{\text {DEEP }}{ }^{45}$ and their corresponding ligand efficiencies $(\eta)^{46}$ are shown in the 5 th and 6 th columns, respectively. The 2 compounds in bold were selected for an in vitro assay because of their availability. Compounds with the same ID have different protonation states. N/A: not applicable.

\begin{tabular}{llrrrr}
\hline \multirow{2}{*}{ ZINC ID } & Receptor & $\begin{array}{r}\text { Tyr433 } \\
\text { contact \# }\end{array}$ & $\begin{array}{r}\Delta \mathbf{G}(\text { Vina) } \\
{[\mathbf{k c a l} / \mathbf{m o l}]}\end{array}$ & $\begin{array}{r}\Delta \mathbf{G}\left(\boldsymbol{K}_{\text {DEEP }}\right) \\
{[\mathbf{k c a l} / \mathbf{m o l}]}\end{array}$ & $\begin{array}{r}\eta\left(\boldsymbol{K}_{\text {DEEP }}\right) \\
{[\mathbf{k c a l} / \mathbf{m o l}]}\end{array}$ \\
\hline $\mathbf{3 1 6 0 7 0 1 8}$ & crystal & $\mathbf{2 5}$ & $\mathbf{- 8 . 9}$ & $-\mathbf{7 . 6 7}$ & $\mathbf{- 0 . 3 2}$ \\
69770999 & crystal & 25 & -8.2 & -8.06 & -0.32 \\
74046068 & crystal & 23 & -8.8 & -8.70 & -0.33 \\
$\mathbf{7 2 2 8 9 8 6 0}$ & crystal & $\mathbf{2 3}$ & $\mathbf{- 8 . 3}$ & $\mathbf{- 8 . 0 1}$ & $\mathbf{- 0 . 3 3}$ \\
24208749 & crystal & 23 & -8.3 & -7.68 & -0.32 \\
72289860 & crystal & 23 & -8.2 & -7.94 & -0.33 \\
27986924 & crystal & 23 & -8.2 & -7.08 & -0.31 \\
21836253 & crystal & 23 & -8.2 & -6.96 & -0.28 \\
29506278 & crystal & 22 & -8.6 & -9.49 & -0.38 \\
89510830 & crystal & 22 & -8.3 & -7.23 & -0.29 \\
78932307 & crystal & 22 & -8.2 & -8.42 & -0.34 \\
12812109 & crystal & 22 & -8.1 & -8.93 & -0.36 \\
56338067 & crystal & 22 & -8.1 & -7.63 & -0.32 \\
89544152 & crystal & 21 & -8.4 & -8.41 & -0.34 \\
72329523 & crystal & 21 & -8.3 & -7.57 & -0.30 \\
80057881 & crystal & 21 & -8.1 & -8.52 & -0.36 \\
32203147 & crystal & 20 & -8.3 & -8.09 & -0.35 \\
85467379 & crystal & 20 & -8.0 & -7.60 & -0.30 \\
\hline Average & N/A & 22 & -8.3 & -8.00 & -0.33 \\
\hline
\end{tabular}




\section{In Vitro Functional Assay}

(a)

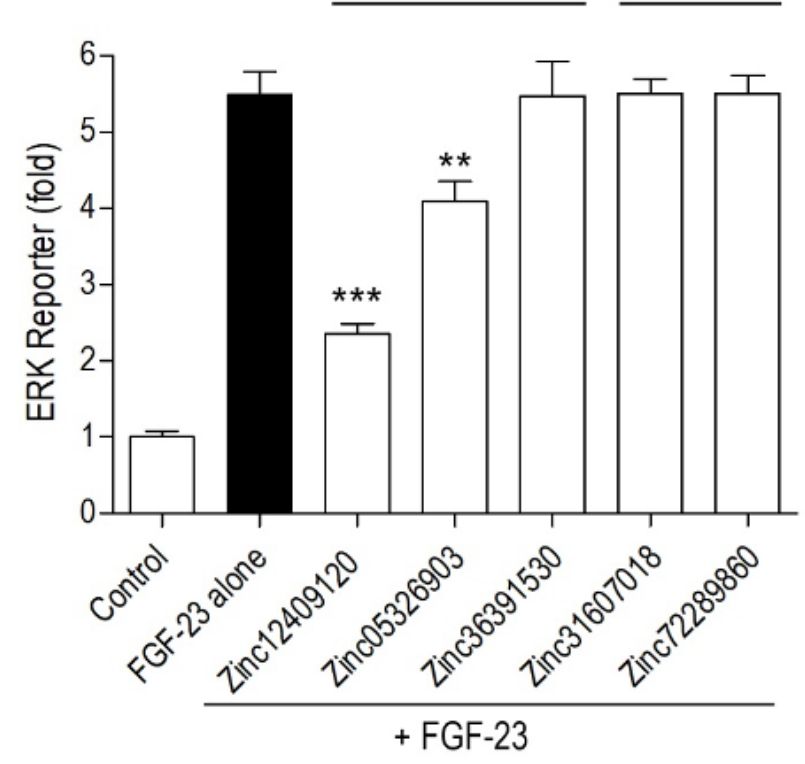

(b)

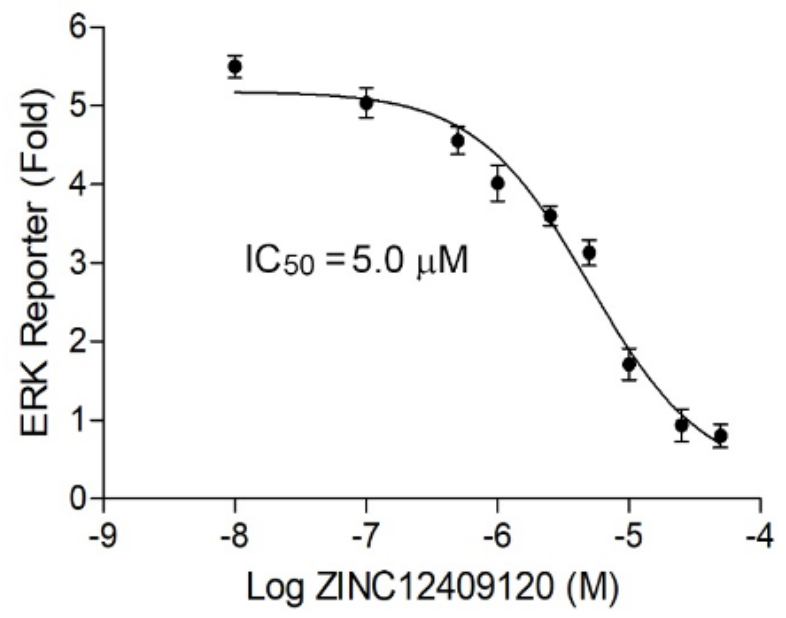

Figure 3. (a) The effects of 5 FGF23-mediated ZINC compounds selected from Tables 3 and 4 and that of FGF23 only (black bar) on ERK reporter activities in $\alpha$-Klotho-transfected HEK 293 T cells. The most left bar shows the effect of $\alpha$-Klotho-transfected HEK 293T cells only as control. Each bar shows average \pm standard deviation among three independent experiments. $* * *$ $(p$-value $\leq 0.001)$ and $* *(p$-value $\leq 0.01)$ indicate statistically significant difference from FGF23 only. (b) Dose-response curve of ZINC12409120.

The ERK is one of the critical pathways for FGF23 signal transduction, and is activated by formation of the FGF23: $\alpha$-Klotho complex in presence of FGFR1c. ${ }^{13,28}$ Reduced ERK activities thus suggest partial disruption or inhibition of the FGF23: $\alpha$-Klotho complex by small molecules. Out of the 5 compounds we tested, ZINC12409120 and ZINC05326903 reduce FGF23-mediated ERK reporter activities by $70 \%$ and $31 \%$ on average, respectively, if FGF23 only and control are considered as full and null activities, respectively (Figure 3(a)). In addition, reduction of ERK activities by each of ZINC12409120 and ZINC05326903 is statistically significant (Figure 3(a)). Further, ZINC12409120 has an $\mathrm{IC}_{50}$ of $5.0 \pm 0.23 \mu \mathrm{M}$ and a smooth dose-response curve (Figure 3(b)). Optimization of this lead compound could potentially result in FGF23: $\alpha$-Klotho interaction inhibitors with sub-micromolar to nanomolar binding affinities to $\alpha$-Klotho. 


\section{In Silico ZINC12409120: $\alpha-K l o t h o$ Interaction Analysis}

To provide guidance on lead optimization of ZINC12409120 in future studies, its two in silico binding poses each on $\alpha$-Klotho cluster $\# 8$ and crystal structure ${ }^{15}$ were analyzed (Figure 4 ). We note that $\Delta \mathrm{G}$ (Vina), $\Delta \mathrm{G}\left(K_{\text {DEEP }}\right)$, and $\eta\left(K_{\text {DEEP }}\right)$ for ZINC12409120 obtained by docking to the crystal structure are $-11.1,-8.9$, and $-0.34 \mathrm{kcal} / \mathrm{mol}$, respectively. ZINC12409120 binds to the KL1-linker-KL2 region in both poses (Figure 4(a)). There is one hydrogen-bond interaction, and no direct contact with a hot spot residue in the pose obtained by ensemble docking to cluster \#8 (Figure 4(b)). The pose from docking to the crystal structure has five hydrogen-bonds to $\alpha$ Klotho (Figure 4(c)), and one of them involves Lys435, a predicted druggable site (See Table 2). In addition, ZINC05326903 also binds to the KL1-linker-KL2 region of $\alpha$-Klotho crystal structure (Figure S4). Further, hot spot analysis on KL1(Glu34-Phe506):KL2(Leu515-Ser950) interface of $\alpha$-Klotho crystal structure shows that Tyr432, a predicted druggable site (See Table 2 ), is a promising hot spot (Table S10).

Taken together, ZINC12409120 does not interact with hot spots residues at the KL1:KL2:FGF23 interface of $\alpha$-Klotho, but has contacts with residues on KL1, the linker, and KL2. This may further hinder FGF23: $\alpha$-Klotho interaction from forming a complex, thereby reducing ERK activities observed in the in vitro assay. Future studies may focus on how to design small molecules with a better geometric fit in between residues on KL1, the linker, and KL2 and contacting Tyr432 on KL1. 
(a)

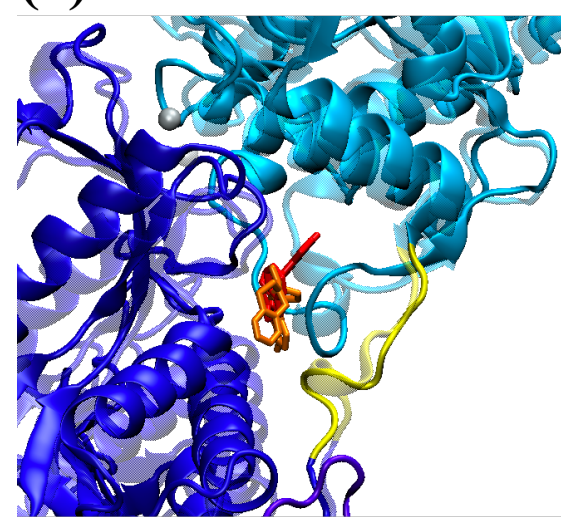

(b)

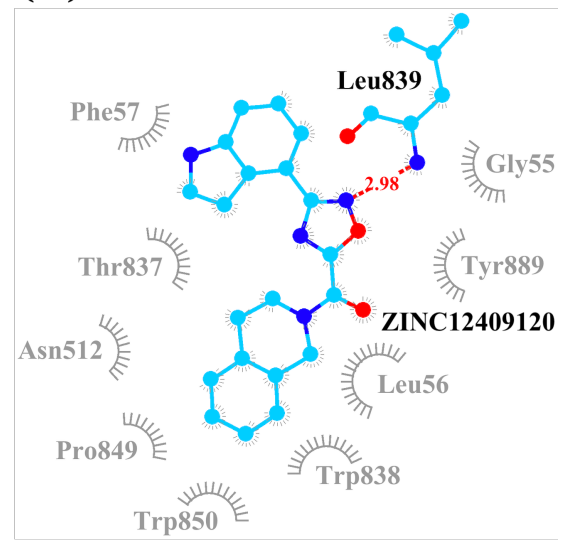

(c)

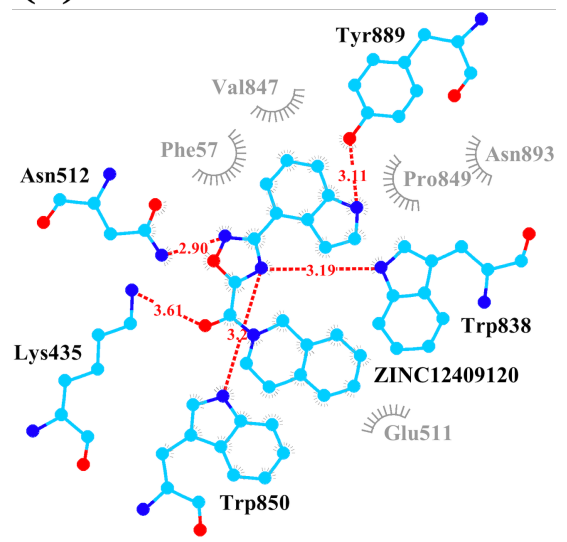

Figure 4. (a) The two in silico binding poses of ZINC12409120 in the KL1-linker-KL2 (light blue-yellow-dark blue) region of $\alpha$-Klotho in ensemble docking. The red and orange ligands bind to $\alpha$-Klotho cluster \#8 (opaque) and crystal structure ${ }^{15}$ (transparent), respectively. (b) The 2D contacting map of ZINC12409120 with $\alpha$-Klotho cluster \#8. (c) The 2D contacting map of ZINC12409120 with $\alpha$-Klotho crystal structure. Hydrogen bonds are shown in red dashed lines with donor-acceptor distances in $\AA$. Hydrophobic interactions are shown in gray. 


\section{Conclusions}

Of the five protein:protein interfaces in the crystal structure of the FGF23:FGFR1c: $\alpha$-Klotho ternary complex (PDB code: 5W21), that between FGF23 and $\alpha$-Klotho is computationally identified as that with the largest fraction (73\%) of hot spots and with two druggable residues on $\alpha$-Klotho. Our hot spot analysis further identified Tyr433 on KL1 domain of $\alpha$-Klotho as a promising binding site for in silico docking. Armed with this information, we screened computationally $\sim 5.5$ million lead-like compounds and identified 23 compounds with the highest predicted protein-ligand binding affinities to $\alpha$-Klotho and another 18 compounds whose binding poses had the largest number of contacts with Tyr433. We re-docked these 41 compounds to the crystal structure and 10 clusters of $\alpha$-Klotho obtained from MD simulations to refine the binding poses, and rescored the binding free energy of the best docking-scored pose for each of the 23 compounds and that of the pose with the largest number of contacts with Tyr433 for each of the 18 compounds. 5 out of 41 compounds were tested in vitro because of their availability. Two of the five compounds significantly reduce FGF23-mediated ERK activity. These two compounds were identified based on the estimated Vina binding affinity, not on contacts with the Tyr433 hot spot. The most potent one (ZINC12409120) reduces the ERK activity by $70 \%$ and has an $\mathrm{IC}_{50}$ of $5.0 \pm 0.23 \mu \mathrm{M}$. Two in silico binding poses of ZINC12409120 on $\alpha$-Klotho from docking reveal that ZINC12409120 is in contact with KL1, KL2, and the linker between KL1 and KL2 domains to plausibly modulate the function of $\alpha$-Klotho. This may disrupt FGF23 binding to $\alpha$-Klotho to reduce ERK activity.

In future studies, ZINC12409120 analogs could be evaluated for binding to $\alpha$-Klotho and efficacy in inhibiting FGF23: $\alpha$-Klotho interactions. Further, the MD clusters of $\alpha$-Klotho may provide functional insights from structural biology and be useful in a variety of ensemble docking tasks. 2 out of 5 compounds tested were found to have functional activity in this study. This illustrates that the computationally relatively inexpensive screening of millions of compounds to a region with druggable sites and large fraction of predicted hot spots may allow small-molecule PPI inhibitors to be identified experimentally even when only a very small number of compounds is tested. In addition, we may perform mutagenesis of $\alpha$-Klotho, test other functions of $\alpha$-Klotho in presence and absence of these 41 compounds, and test compounds in a bioluminescence resonance energy transfer (BRET) assay for target engagement. 


\section{Supporting Information Available}

S1. MD Simulations of $\alpha$-Klotho; S2. Clustering of $\alpha$-Klotho; S3. Docking to $\alpha$-Klotho; S4. Hot Spot Analysis on FGF23:FGFR1c: $\alpha-K 1$ tho Ternary Complex; S5. Contact Analysis on the 41 Compounds; S6. In Silico ZINC05326903: $\alpha$-Klotho Interaction Analysis; S7. Hot Spot Analysis on KL1:KL2 Interface of $\alpha$-Klotho; alpha-Klotho.zip; ZINC-Compound.csv

\section{Acknowledgement}

This research was funded by the Laboratory Directed Research and Development Program of Oak Ridge National Laboratory. L. D. Q. was supported by grant R01-DK121132 of National Institutes of Health. This research used resources of the Compute and Data Environment for Science (CADES) at the Oak Ridge National Laboratory, which is supported by the Office of Science of the U.S. Department of Energy under Contract No. DE-AC05-00OR22725.

\section{References}

(1) Quarles, L. D. The Bone and Beyond: 'Dem Bones' Are Made for More Than Walking. Nat. Med. 2011, 17, 428-430.

(2) Quarles, L. D. Evidence for a Bone-Kidney Axis Regulating Phosphate Homeostasis. J. Clin. Invest. 2003, 112, 642-646.

(3) Weber, T. J.; Liu, S.; Indridason, O. S.; Quarles, L. D. Serum FGF23 Levels in Normal and Disordered Phosphorus Homeostasis. J. Bone Miner. Res. 2003, 18, 1227-1234.

(4) Sancho, P. A. Complications of Phosphate and Vitamin D Treatment in X-Linked Hypophosphataemia. Adv. Ther. 2020, 37, 105-112.

(5) de Paula Colares Neto, G.; Yamauchi, F. I.; Baroni, R. H.; de Andrade Bianchi, M.; Gomes, A. C.; Chammas, M. C.; Martin, R. M. Nephrocalcinosis and Nephrolithiasis in X-Linked Hypophosphatemic Rickets: Diagnostic Imaging and Risk Factors. J. Endocrine Soc. 2019, 3, 1053-1061.

(6) Florenzano, P.; Gafni, R. I.; Collins, M. T. Tumor-Induced Osteomalacia. Bone Rep. 2017, 7, 90-97.

(7) Fukumoto, S. Diagnostic Modalities for FGF23-Producing Tumors in Patients with TumorInduced Osteomalacia. Endocrinol. Metab. 2014, 29, 136-143. 
(8) Carpenter, T. O.; Whyte, M. P.; Imel, E. A.; Boot, A. M.; Högler, W.; Linglart, A.; Padidela, R.; van’t Hoff, W.; Mao, M.; Chen, C.-Y.; Skrinar, A.; Kakkis, E.; Martin, J. S.; Portale, A. A. Burosumab Therapy in Children with X-Linked Hypophosphatemia. N. Engl. J. Med. 2018, 378, 1987-1998.

(9) Lamb, Y. N. Burosumab: First Global Approval. Drugs 2018, 78, 707-714.

(10) Carpenter, T. O.; Imel, E. A.; Ruppe, M. D.; Weber, T. J.; Klausner, M. A.; Wooddell, M. M.; Kawakami, T.; Ito, T.; Zhang, X.; Humphrey, J.; Insogna, K. L.; Peacock, M. Randomized Trial of the Anti-FGF23 Antibody KRN23 in X-Linked Hypophosphatemia. J. Clin. Invest. 2014, 124, 1587-1597.

(11) Zhang, X.; Imel, E. A.; Ruppe, M. D.; Weber, T. J.; Klausner, M. A.; Ito, T.; Vergeire, M.; Humphrey, J.; Glorieux, F. H.; Portale, A. A.; Insogna, K.; Carpenter, T. O.; Peacock, M. Pharmacokinetics and Pharmacodynamics of a Human Monoclonal Anti-FGF23 Antibody (KRN23) in the First Multiple Ascending-Dose Trial Treating Adults With X-Linked Hypophosphatemia. J. Clin. Pharmacol. 2016, 56, 176-185.

(12) Goetz, R.; Beenken, A.; Ibrahimi, O. A.; Kalinina, J.; Olsen, S. K.; Eliseenkova, A. V.; Xu, C.F.; Neubert, T. A.; Zhang, F.; Linhardt, R. J.; Yu, X.; White, K. E.; Inagaki, T.; Kliewer, S. A.; Yamamoto, M.; Kurosu, H.; Ogawa, Y.; Kuro-o, M.; Lanske, B.; Razzaque, M. S.; Mohammadi, M. Molecular Insights into the Klotho-Dependent, Endocrine Mode of Action of Fibroblast Growth Factor 19 Subfamily Members. Mol. Cell. Biol. 2007, 27, 3417-3428.

(13) Xiao, Z.; Riccardi, D.; Velazquez, H. A.; Chin, A. L.; Yates, C. R.; Carrick, J. D.; Smith, J. C.; Baudry, J.; Quarles, L. D. A Computationally Identified Compound Antagonizes Excess FGF-23 Signaling in Renal Tubules and a Mouse Model of Hypophosphatemia. Sci. Signal. 2016, 9, ra113.

(14) Xiao, Z.; Liu, J.; Liu, S.-H.; Petridis, L.; Cai, C.; Cao, L.; Wang, G.; Chin, A. L.; Cleveland, J. W.; Ikedionwu, M. O.; Carrick, J. D.; Smith, J. C.; Quarles, L. D. Small Molecule FGF23 Inhibitors Increase Serum Phosphate and Improve Skeletal Abnormalities in Hyp Mice. bioRxiv Preprint 2020, DOI: 10.1101/2020.08.04.236877.

(15) Chen, G.; Liu, Y.; Goetz, R.; Fu, L.; Jayaraman, S.; Hu, M.-C.; Moe, O. W.; Liang, G.; Li, X.; Mohammadi, M. $\alpha$-Klotho is a Non-Enzymatic Molecular Scaffold for FGF23 Hormone Signaling. Nature 2018, 553, 461-466. 
(16) Damm-Ganamet, K. L.; Arora, N.; Becart, S.; Edwards, J. P.; Lebsack, A. D.; McAllister, H. M.; Nelen, M. I.; Rao, N. L.; Westover, L.; Wiener, J. J. M.; Mirzadegan, T. Accelerating Lead Identification by High Throughput Virtual Screening: Prospective Case Studies from the Pharmaceutical Industry. J. Chem. Inf. Model. 2019, 59, 2046-2062.

(17) Wingert, B. M.; Camacho, C. J. Improving Small Molecule Virtual Screening Strategies for the Next Generation of Therapeutics. Curr. Opin. Chem. Biol. 2018, 44, 87-92.

(18) Macalino, S. J. Y.; Basith, S.; Clavio, N. A. B.; Chang, H.; Kang, S.; Choi, S. Evolution of In Silico Strategies for Protein-Protein Interaction Drug Discovery. Molecules 2018, 23, 1963.

(19) Wang, H.; Liu, C.; Deng, L. Enhanced Prediction of Hot Spots at Protein-Protein Interfaces Using Extreme Gradient Boosting. Sci. Rep. 2018, 8, 14285.

(20) Xia, J.; Yue, Z.; Di, Y.; Zhu, X.; Zheng, C.-H. Predicting Hot Spots in Protein Interfaces

Based on Protrusion Index, Pseudo Hydrophobicity and Electron-Ion Interaction Pseudopotential Features. Oncotarget 2016, 7, 18065-18075.

(21) Deng, L.; Guan, J.; Wei, X.; Yi, Y.; Zhang, Q. C.; Zhou, S. Boosting Prediction Performance of Protein-Protein Interaction Hot Spots by Using Structural Neighborhood Properties. J. Comput. Biol. 2013, 20, 878-891.

(22) Xia, J.-F.; Zhao, X.-M.; Song, J.; Huang, D.-S. APIS: Accurate Prediction of Hot Spots in Protein Interfaces by Combining Protrusion Index with Solvent Accessibility. BMC Bioinformatics 2010, 11, 174.

(23) Cho, K.-i.; Kim, D.; Lee, D. A Feature-Based Approach to Modeling Protein-Protein Interaction Hot Spots. Nucleic Acids Res. 2009, 37, 2672-2687.

(24) Kortemme, T.; Baker, D. A Simple Physical Model for Binding Energy Hot Spots in Protein-Protein Complexes. Proc. Natl. Acad. Sci. U. S. A. 2002, 99, 14116-14121.

(25) Kozakov, D.; Grove, L. E.; Hall, D. R.; Bohnuud, T.; Mottarella, S. E.; Luo, L.; Xia, B.; Beglov, D.; Vajda, S. The FTMap Family of Web Servers for Determining and Characterizing Ligand-Binding Hot Spots of Proteins. Nat. Protoc. 2015, 10, 733-755.

(26) Zhu, X.; Mitchell, J. C. KFC2: A Knowledge-Based Hot Spot Prediction Method Based on Interface Solvation, Atomic Density, and Plasticity Features. Proteins 2011, 79, 2671-2683. (27) Darnell, S. J.; Page, D.; Mitchell, J. C. An Automated Decision-Tree Approach to Predicting Protein Interaction Hot Spots. Proteins 2007, 68, 813-823. 
(28) Urakawa, I.; Yamazaki, Y.; Shimada, T.; Iijima, K.; Hasegawa, H.; Okawa, K.; Fujita, T.; Fukumoto, S.; Yamashita, T. Klotho Converts Canonical FGF Receptor into a Specific Receptor for FGF23. Nature 2006, 444, 770-774.

(29) Webb, B.; Sali, A. Comparative Protein Structure Modeling Using MODELLER. Curr. Protoc. Bioinform. 2016, 54, 5.6.1-5.6.37.

(30) Humphrey, W.; Dalke, A.; Schulten, K. VMD: Visual Molecular Dynamics. J. Mol. Graph. 1996, 14, 33-38.

(31) Huang, J.; Rauscher, S.; Nawrocki, G.; Ran, T.; Feig, M.; de Groot, B. L.; Grubmüller, H.; MacKerell Jr., A. D. CHARMM36m: An Improved Force Field for Folded and Intrinsically Disordered Proteins. Nat. Methods 2017, 14, 71-73.

(32) Best, R. B.; Zhu, X.; Shim, J.; Lopes, P. E. M.; Mittal, J.; Feig, M.; MacKerell Jr., A. D. Optimization of the Additive CHARMM All-Atom Protein Force Field Targeting Improved Sampling of the Backbone $\phi, \psi$ and Side-Chain $\chi_{1}$ and $\chi_{2}$ Dihedral Angles. J. Chem. Theory Comput. 2012, 8, 3257-3273.

(33) Abraham, M. J.; Murtola, T.; Schulz, R.; Páll, S.; Smith, J. C.; Hess, B.; Lindahl, E. GROMACS: High Performance Molecular Simulations Through Multi-Level Parallelism from Laptops to Supercomputers. SoftwareX 2015, 1-2, 19-25.

(34) Páll, S.; Abraham, M. J.; Kutzner, C.; Hess, B.; Lindahl, E. Tackling Exascale Software Challenges in Molecular Dynamics Simulations with GROMACS. Lecture Notes in Computer Science 2015, 8759, 3-27.

(35) Morris, G. M.; Huey, R.; Lindstrom, W.; Sanner, M. F.; Belew, R. K.; Goodsell, D. S.; Olson, A. J. AutoDock4 and AutoDockTools4: Automated Docking with Selective Receptor Flexibility. J. Comput. Chem. 2009, 30, 2785-2791.

(36) Amaro, R. E.; Baudry, J.; Chodera, J.; Demir, O.; McCammon, J. A.; Miao, Y.; Smith, J. C. Ensemble Docking in Drug Discovery. Biophys. J. 2018, 114, 2271-2278.

(37) Jorgensen, W. L.; Chandrasekhar, J.; Madura, J. D.; Impey, R. W.; Klein, M. L. Comparison of Simple Potential Functions for Simulating Liquid Water. J. Chem. Phys. 1983, 79, 926-935. (38) Daura, X.; Gademann, K.; Jaun, B.; Seebach, D.; van Gunsteren, W. F.; Mark, A. E. Peptide Folding: When Simulation Meets Experiment. Angew. Chem.-Int. Edit. 1999, 38, 236-240.

(39) Ellingson, S. R.; Miao, Y.; Baudry, J.; Smith, J. C. Multi-Conformer Ensemble Docking to Difficult Protein Targets. J. Phys. Chem. B 2015, 119, 1026-1034. 
(40) Teague, S. J.; Davis, A. M.; Leeson, P. D.; Oprea, T. The Design of Leadlike Combinatorial Libraries. Angew. Chem.-Int. Edit. 1999, 38, 3743-3748.

(41) Irwin, J. J.; Sterling, T.; Mysinger, M. M.; Bolstad, E. S.; Coleman, R. G. ZINC: A Free Tool to Discover Chemistry for Biology. J. Chem. Inf. Model. 2012, 52, 1757-1768.

(42) Lyu, J.; Wang, S.; Balius, T. E.; Singh, I.; Levit, A.; Moroz, Y. S.; O’Meara, M. J.; Che, T.; Algaa, E.; Tolmachova, K.; Tolmachev, A. A.; Shoichet, B. K.; Roth, B. L.; Irwin, J. J. UltraLarge Library Docking for Discovering New Chemotypes. Nature 2019, 566, 224-229.

(43) Ellingson, S. R.; Smith, J. C.; Baudry, J. VinaMPI: Facilitating Multiple Receptor HighThroughput Virtual Docking on High-Performance Computers. J. Comput. Chem. 2013, 34, 2212-2221.

(44) Trott, O.; Olson, A. J. AutoDock Vina: Improving the Speed and Accuracy of Docking with a New Scoring Function, Efficient Optimization, and Multithreading. J. Comput. Chem. 2010, $31,455-461$.

(45) Jiménez, J.; Škalič, M.; Martínez-Rosell, G.; De Fabritiis, G. KDEEP: Protein-Ligand Absolute Binding Affinity Prediction via 3D-Convolutional Neural Networks. J. Chem. Inf. Model. 2018, 58, 287-296.

(46) Hopkins, A. L.; Groom, C. R.; Alex, A. Ligand Efficiency: A Useful Metric for Lead Selection. Drug Discov. Today 2004, 9, 430-431.

(47) Laskowski, R. A.; Swindells, M. B. LigPlot+: Multiple Ligand-Protein Interaction Diagrams for Drug Discovery. J. Chem. Inf. Model. 2011, 51, 2778-2786.

(48) Ran, X.; Gestwicki, J. E. Inhibitors of Protein-Protein Interactions (PPIs): An Analysis of Scaffold Choices and Buried Surface Area. Curr. Opin. Chem. Biol. 2018, 44, 75-86. (49) Daina, A.; Michielin, O.; Zoete, V. SwissADME: A Free Web Tool to Evaluate Pharmacokinetics, Drug-Likeness and Medicinal Chemistry Friendliness of Small Molecules. Sci. Rep. 2017, 7, 42717.

(50) Baell, J. B.; Holloway, G. A. New Substructure Filters for Removal of Pan Assay Interference Compounds (PAINS) from Screening Libraries and for Their Exclusion in Bioassays. J. Med. Chem. 2010, 53, 2719-2740. 